\title{
Time resolved spectroscopy of Balloon 090100001
}

\author{
R. $\varnothing$ stensen, ${ }^{1}$ J. Telting, ${ }^{2}$ U. Heber ${ }^{3}$ \\ ${ }^{1}$ Instituut voor Sterrenkunde, Leuven, Belgium \\ ${ }^{2}$ Nordic Optical Telescope, La Palma, Spain \\ ${ }^{3}$ Dr. Remeis-Sternwarte, Bamberg, Germany
}

\begin{abstract}
We obtained 2552 good low-resolution spectra of the bright sdBV star Balloon 090100001 with the Nordic Optical Telescope in August/September 2004. Results of the frequency analysis of this dataset have already been published (Telting \& Østensen 2006; TØ06). Eight independent frequencies were recovered in radial velocity and equivalent width, all in agreement with established photometric pulsation frequencies (Baran et al. 2006). The radial velocity amplitude of the main mode was found to be $18.9 \mathrm{~km} / \mathrm{s}$ which is the largest radialvelocity amplitude found in a pulsating $\mathrm{sdB}$ star. Here we report our preliminary results from phase folding and fitting the spectroscopic data to synthetic model grids.
\end{abstract}

\section{Results and analysis}

Our spectra cover the wavelength range $3500-5050 \AA$ with a spectral resolution of $\sim 3 \AA$ at a dispersion of $0.77 \AA / \mathrm{px}$. Each exposure was $30 \mathrm{~s}$. In order to derive physical parameters, we fit the spectroscopic data to an LTE hydrogen + helium model grid suitable for sdB stars in this temperature range (Heber et al. 2000). The normalized and detrended spectra were folded into 20 phase bins on the main pulsation period, with the first bin centered on the phase given in Table 7 of TØ06. The physical parameters derived from this procedure is shown in Fig. 1. A sinusoidal fit (dotted line) to the points gives: $T_{\text {eff }}=28883 \pm 1186 \pm 23 \mathrm{~K}$, $\log g=5.416 \pm 0.084 \pm 0.002$ and $\log y=-2.730 \pm 0.003$. The values quoted for $T_{\text {eff }}$ and $\log g$ are the mean, amplitude and fitting error of the sinusoidal fit, and for $\log y$ just the rms error when fitting a constant.

The amplitude of the radius variation due to the $18.9 \mathrm{~km} / \mathrm{s}$ velocity amplitude of the main mode is $A_{r}=A_{v} P / 2=1072 \mathrm{~km}$; the corresponding acceleration is $A_{a}=A_{v} 2 / P=333 \mathrm{~m} / \mathrm{s}^{2}$. At the observed mean gravity this corresponds to $\log g=0.056$ dex. The change in gravity due to the change in radius is only $\log g=0.006$ dex. Together these come close to the observed gravity variation of $\log g=0.084 \mathrm{dex}$, but the discrepancy is significant. Due to the high amplitude of the main mode and the complex shape of the window function of our observations (see Fig. 3 in ТØ06), the second, third and fourth highest peak in the radial velocity spectrum are all strongly contaminated by the main mode, and are only recovered in the Fourier transform after prewhitening. Phase folding of the spectra on the secondary pulsation frequencies can therefore not produce anything useful, unless we first prewhiten the main mode from the spectra. We have implemented a procedure that generates a synthetic spectrum of the main pulsation mode for each spectroscopic observation, and subtracts the difference between the model for the time of observation and a model for the mean parameters, before folding the spectra on the secondary frequencies. This procedure gives useful results for the second highest peak in the velocity spectrum (see Fig. 1). The fourth peak is also recovered using this procedure, but we still have problems with the third peak. 

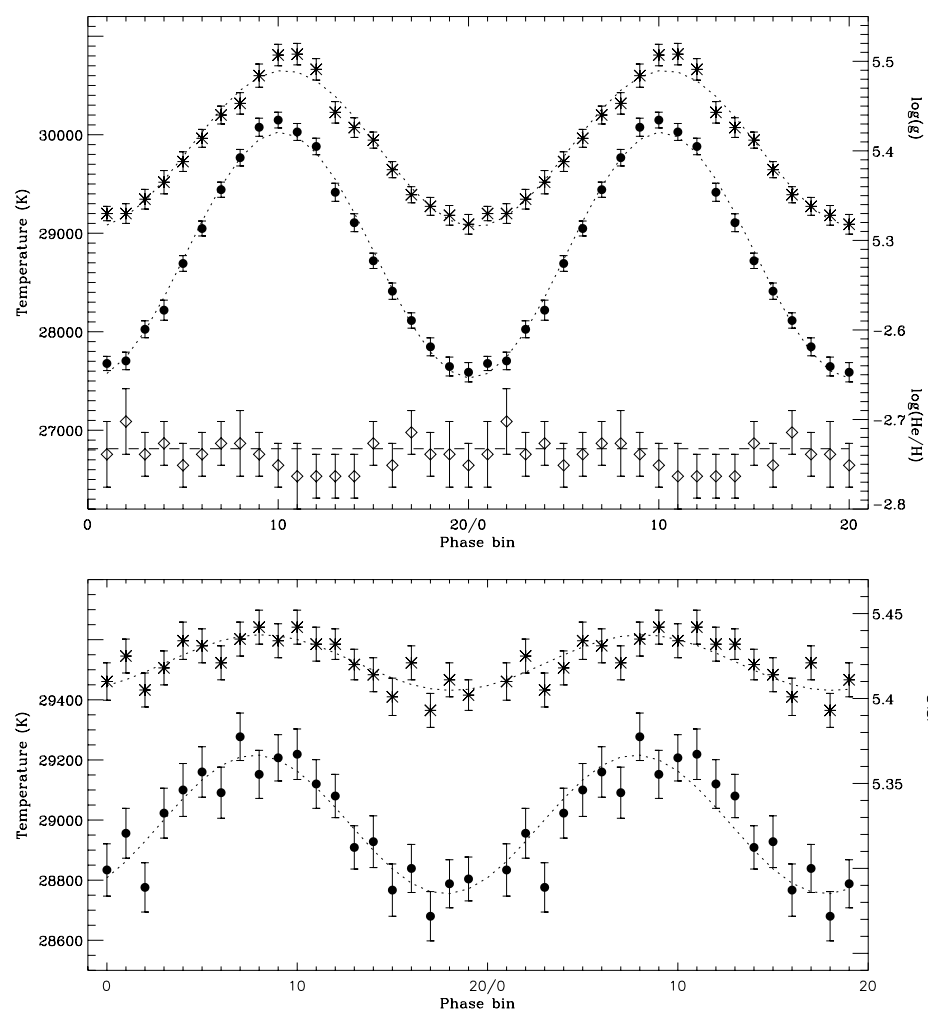

Figure 1: Upper panel: Spectroscopic model fit to the 20 spectra binned on the main pulsation period. The upper curve is the gravity, the middle shows effective temperature and the lower curve the Helium abundance. Note that the temperature and gravity were refitted, after fixing the Helium abundance to the mean value. Lower panel: Same, but for the spectra folded on the second highest pulsation frequency, and after spectral prewhitening of the main mode.

\section{References}

Baran A., Oreiro R., Pigulski A., Pérez Hernández F., Ulla A., 2006, Baltic Astron., 15, 227

Telting J. H., Østensen R., 2006, A\&A, 450, 1149

Heber U., Reid I. N., Werner K., 2000, A\&A, 363, 198 